\title{
FILLET OPERATIONS WITH RECURSIVE SUBDIVISION SURFACES
}

\author{
Zheng XU \\ Dept.of Information and Computer Sciences, SAITAMA University \\ xuz@ke.ics.saitama-u.ac.jp \\ Kunio KONDO \\ Dept.of Information and Computer Sciences, SAITAMA University \\ kondo@ke.ics.saitama-u.ac.jp
}

\begin{abstract}
Recursively subdividing polyhedral networks, often called polyhedral subdivision, has become one of the basic tools in Computer Aided Geometric Design(CAGD) for modeling complex surfaces. In this paper, we propose a set of rules for making fillet operations on polyhedral networks. The fillet operations are implemented by subdividing a polyhedral network one step with given fillet values of edges of the polyhedral network. After fillet operations, we tried two basic recursive subdivision methods Doo-Sabin and Catmull-Clark to generate surfaces. Generally, the number of edges that meet at one vertex is no restriction and the fillet values of every edge can be different. The fillet operations proposed here will strengthen the functions of Computer Graphics systems which use subdivision surfaces to model surfaces.
\end{abstract}

Keywords: Recursive Subdivision Surfaces, Doo-Sabin surface, Catmull-Clark surface, B-Spline Curves, Fillet Operations

\section{INTRODUCTION}

\subsection{BACKGROUND}

As polyhedral subdivision process provides a simple and efficient way to generate surfaces over polyhedral networks, it has been interested by a lot of researchers since the first two methods were proposed by E.E.Catmull, J.H.Clark[2] and D.Doo, M.Sabin[3] in 1978. A lot of approaches were made or are being made to follow or extend polyhedral subdivision method. Nasri extended Doo-Sabin method to generate a surface which interpolates some or all vertices of a polyhedron[4],More- 
over, he proposed an algorithm to generate subdivision surfaces which interpolate B-spline curves[5][6]. J.Peters[9] extended the subdivision technology to generate surfaces by separating singular regions after a few steps of Doo-Sabin subdivision. M.Halstead et al.[10] proposed an interpolation method using Catmull-Clark surfaces. N.Dyn, D.Levin and J.Gregory[8] proposed a butterfly subdivision scheme. U. Reif[7] gave a serious analysis on the smoothness properties of subdivision surfaces such as Doo-Sabin and Catmull-Clark surfaces. Recently the success of CG animation system using subdivision surfaces developed by Pixar Animation Studios[11] showed the recursive subdivision surfaces will be an essential role in modeling surfaces.

\subsection{DOO-SABIN AND CATMULL-CLARK SUBDIVISION SURFACES}

As in our approach, the surfaces are generated by Doo-Sabin and Catmull-Clark subdivision methods, we overview these two methods in this section.

1.2.1 Chaikin's algorithm. The fundamental idea of both Doo-Sabin surfaces and Catmull-Clark surfaces goes back to Chaikin's [1] algorithm which generates a quadratic B-spline curve from a polygon by successively cutting its corners. Each subdivision generates two new points on each polygon leg at $(1 / 4,3 / 4)$. For a polygon with $n$ vertices $\left(V_{i}\right)_{1 \leq i \leq n}$, on polygon leg $\left(V_{i}, V_{i+1}\right)$, two new points are given by the following:

$$
V_{i}^{\prime \prime}=\frac{3}{4} V_{i}+\frac{1}{4} V_{i+1} . \quad V_{i+1}^{\prime}=\frac{1}{4} V_{i}+\frac{3}{4} V_{i+1} .
$$

Then the new polygon is linked by the new generated points $V_{i}^{\prime}, V_{i}^{\prime \prime}$ corresponding to $\left(V_{i}\right)_{1 \leq i \leq n}$. Repeat the subdivision process, we can get a quadratic B-spline curve.

\subsubsection{Doo-Sabin and Catmull-Clark subdivision method.}

In 1978, D.Doo, M.Sabin[3] applied Chaikin's idea to generating surfaces. In Doo-Sabin's method, surfaces are generated from polyhedral networks by successively cutting polyhedron's corners and edges. 'The algorithm can be described as follows and illustrated in Fig.1, some terms proposed by Nasri[4] are used hereunder:

1 For every vertex $V_{i}$ of the polyhedron $P_{i}$, a new vertex $V_{i}^{\prime}$, termed image, is generated on each face adjacent to $V_{i}$. 
2 For each face $F_{i}$ of $P_{i}$, a new face, termed $F$-face, is made by connecting the images, the vertices $V_{i}^{\prime}$ s generated in step 1 .

3 For each edge $E_{i}$ common to two faces $F_{i}$ and $F_{i}^{\prime}$, a new 4 -sided face, termed $E$-face, is made by connecting the images of the end vertices of $E_{i}$ on the faces $F_{i}$ and $F_{i}^{\prime}$.

4 For each vertex $V_{i}$, where $n$ faces meet, a new face, termed $V$-face, is made by connecting the images of $V_{i}$ on the faces meeting at $V_{i}$.

The image vertex $V_{i}^{\prime}$ generated in step 1 is functions only of the vertices of $P_{i}$. That is :

$$
V_{i}^{\prime}=\sum_{j=1}^{n} a_{i j} V_{j}
$$

Where $V_{j}$ are the vertices of the old faces and $V_{i}^{\prime}$ is the new vertex of $V_{j}$, and $a_{i j}$ are weights.

$$
\begin{gathered}
a_{i j}=\frac{n+5}{4 n} \quad \text { for } i=j, \\
a_{i j}=\frac{3+2 \cos \left(2 \pi\left(\frac{i-j}{n}\right)\right)}{4 n} \quad \text { for } i \neq j .
\end{gathered}
$$

Fig.1(a) illustrates the $F$-face, E-face and $V$-face of Doo-Sabin's subdivision method. The face of a polyhedron is closed in solid nodes and generated $F$-face, E-face and $V$-face are closed in hollow nodes. Fig.1(b)(e) illustrate a cube and make Doo-Sabin's subdivision process over it. Doo-Sabin surfaces are composed of biquadratic splines. Using similar idea, Catmull and Clark implemented a cubic spline surface simultaneously in 1978. Fig.2 illustrates a Catmull-Clark surface generated from a cube.

\subsection{MOTIVATIONS}

Surfaces can be generated easily by subdividing polyhedral networks recursively, but complex or precise polyhedral networks have to be defined. For example, for getting a cylinder-like shape (Fig.3(d)) with Doo-Sabin subdivision method, a polyhedron shown in Fig.3(b) has to be defined. we can not get cylinder-like shape by subdividing a simple cube (Fig.3(a)). Over simple or rough polyhedral network, it is difficult to get the surfaces which designers want to model. According to the subdivision method(Doo-Sabin, Catmull-Clark etc.), special polyhedral networks have to be defined. Obviously, it is troublesome and inefficient. So for a system that uses subdivision method to generate surfaces, some 


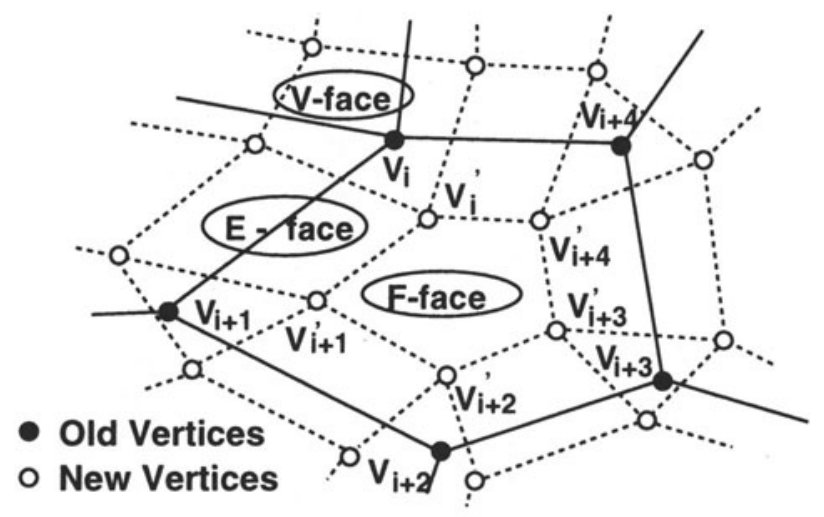

(a)

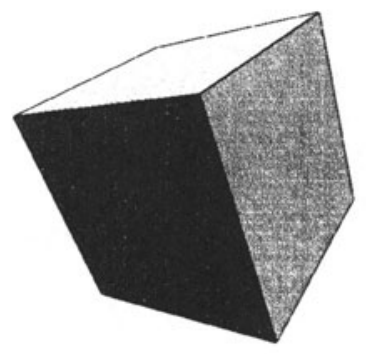

(b)

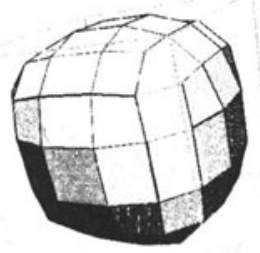

(d)

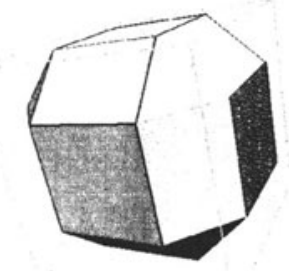

(c)

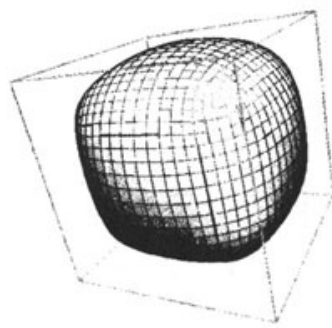

(e)

Figure 1 Doo-Sabin's method.(a) Three types of faces.(b)A cube. (c)The cube and its first subdivision.(d)The cube and its second subdivision. (e)The cube and its fourth subdivision. 


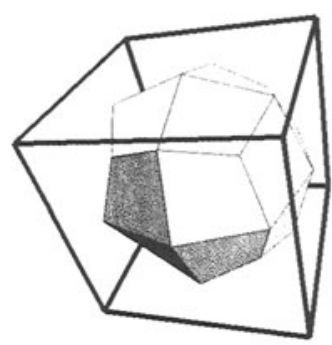

(a)

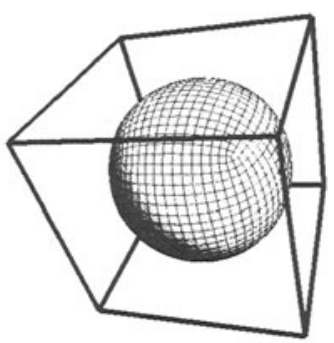

(b)

Figure 2 Catmull-Clark's method.(a)The cube and its first subdivision. (b)The cube and its fourth subdivision.

operations have to be developed for supporting the subdivision method. In this paper, we propose a set of rules for implementing fillet operations , an essential process in design systems. In our method, according to fillet values of every edge of polyhedral networks, the initial polyhedral networks are subdivided one step using the rules proposed here, and then Doo-Sabin subdivision or Catmull-Clark process is carried out over the modified polyhedral networks. The number of edges that meet at one vertex is no restriction and the fillet values of every edge can be different.

\section{THE RULES OF SUBDIVISION IN FILLET OPERATIONS}

\subsection{DEFINITIONS AND REGULAR PROCESS}

Before giving our rules, some terms should be defined. First is the fillet value. It describes the sharpness of an edge and is defined as the distance between an edge and a new parallel line, termed fillet line, on the faces that the edge is common to. Each edge has two fillet values and two fillet lines. The two fillet values can be different. The fillet value of an edge also can be 0 . Now we assume the fillet value is not equal to 0. Similar to Doo-Sabin subdivision method, the fillet operations will subdivide the initial polyhedral networks and generate new faces $F$-face, E-face and $V$-face according to initial polyhedra's faces, edges and vertices. For describing our method smoothly, first, we make fillet operations on a cube with same fillet value for all edges. The fillet process can be described as follow steps (Referring to Fig.4): 


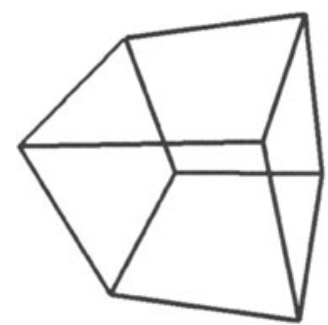

(a)

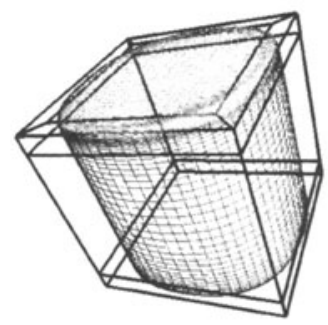

(c)

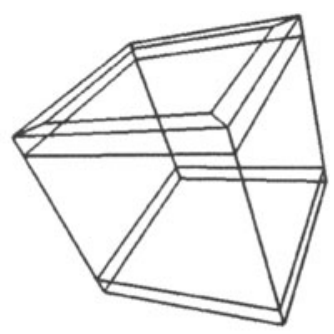

(b)

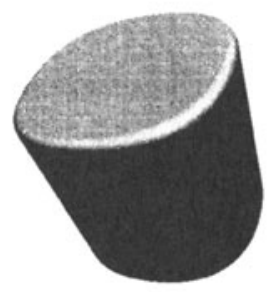

(d)

Figure 3 A Cylinder-like shape generated by Doo-Sabin subdivision method. (a) A Cube.(b)The modified cube.(c)The cube and its third subdivision. (d)The fourth subdivision.

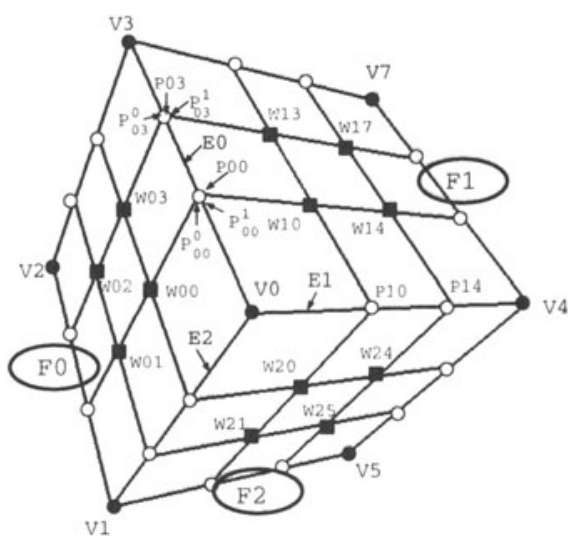

Figure 4 The regular subdivision of fillet operations. Solid circle nodes are the vertices of the cube, hollow circle nodes are the fillet edge points. Solid square nodes are fillet face points. 


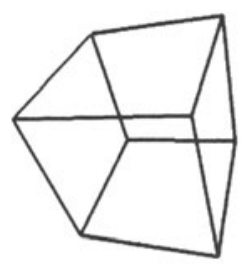

(a)

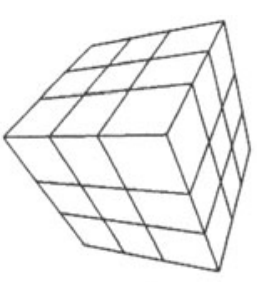

(b)

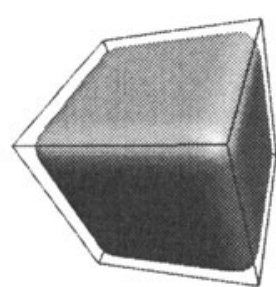

Figure 5 The regular fillet operation on a cube. (a) A Cube.(b)The modified cube after fillet operations. (c)The fourth Doo-Sabin subdivision over modified cube.

1 For each vertex $V_{i}$ on a face $F_{s}$, an intersection point of two fillet lines, corresponding to the two contiguous edges that have common end point $V_{i}$, is generated. The intersection point called fillet face point termed $W_{s i}$ (Solid square nodes in Fig.4).

2 For each edge $E_{i}$ linking vertex $V_{k}, V_{j}$ and shared by face $F_{s}, F_{t}$, four intersection points of the $E_{i}$ and fillet lines on the two faces $F_{s}, F_{t}$ are generated, respectively. They are called fillet edge points, termed $P_{i j}^{s}, P_{i j}^{t}, P_{i k}^{s}$ and $P_{i k}^{t}$ (Hollow circle nodes in Fig.4). Corresponding to $V_{j}$, there are two points $P_{i j}^{s}$ and $P_{i j}^{t}$ are generated on edge $E_{i}$, but only one point between $P_{i j}^{s}$ and $P_{i j}^{t}$ will be used to generate new faces, the useful point is termed $P_{i j}$. Similarly corresponding to $V_{k}$, the useful point is $P_{i k}$. Here, the three points $P_{i j}^{s}, P_{i j}^{t}$ and $P_{i j}$ are same point. Three points $P_{i k}^{s}, P_{i k}^{t}$ and $P_{i k}$ are same point too. For example, in Fig. $4, P_{00}^{1}, P_{00}^{0}$ and $P_{00}$ are same point.

3 For every face $F_{i}$, a new face, termed as $(F-f a c e)_{i}$ is generated by linking all fillet face points $W_{i j}$ (Solid square nodes in Fig.4) on the face face $F_{i}$.

4 For each edge $E_{i}$ linking vertices $V_{j}, V_{k}$, common to two faces $F_{s}$, $F_{t}$, two new 4-sided faces, termed $(E-\text { face })_{s i}$ and $(E-f a c e)_{t i}$ are generated by linking $\left(W_{s j}, W_{s k}, P_{i k}, P_{i j}\right)$ and $\left(W_{t j}, W_{t k}, P_{i k}, P_{i j}\right)$, respectively.

5 For each vertex $V_{i}$, a 4 -sided face termed $(V-f a c e)_{j i}$, is generated on every face $F_{j}$ that $V_{i}$ is common to. It is linked by $V_{i}$, the fillet face point $W_{j i}$ and two fillet edge points. For example, in Fig.4 the $(V-\text { face })_{00}$ is linked by $\left(V_{0}, P_{00}, W_{00}, P_{20}\right)$. 


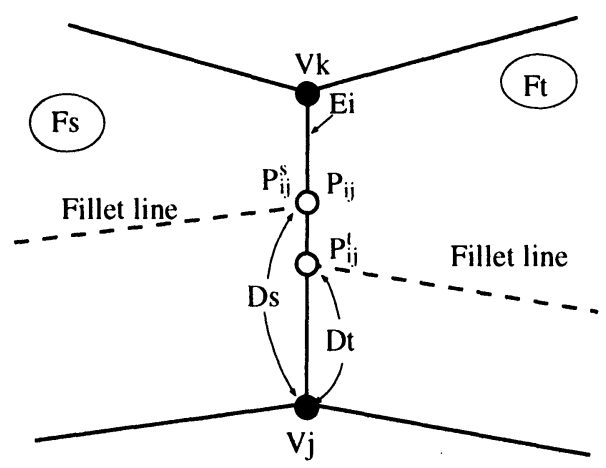

Figure 6 Rule 1. Fillet values are not equal each other. Solid circle nodes are vertices of the polyhedron, hollow circle nodes are fillet edge points.

After subdividing the cube according to above steps, subdivision process will be carried out recursively. Fig. 5 illustrates the procedure of making fillet operations on a cube with Doo-Sabin surface.

\subsection{RULE 1}

The above process is a special case. In general, the fillet values of the edges of a polyhedron are not equal each other. Here we give the rule 1. Referring to Fig.6, for every edge $E_{i}$ linking vertices $\left(V_{k}, V_{j}\right)$, common to faces $F_{s}$ and $F_{t}$, the fillet values, distances between $P_{i j}^{s}$ and $V_{j}, P_{i j}^{t}$ and $V_{j}$, are $D s$ and $D t$, respectively. If $D s$ is larger than $D t, P_{i j}^{s}$ will be treated as useful fillet edge point $P_{i j}$. Here the $V_{j}$ must be a convex point on both $F_{s}$ and $F_{t}$.

\subsection{RULE 2}

Referring to Fig. 7, for a face $F_{s}$ and a vertex $V_{i}$ of $F_{s}$, two edges of $F_{s}$ having common end point $V_{i}$ are $E_{j}$ and $E_{k}$. Corresponding to $E_{j}$ and $E_{k}$, the two fillet lines on $F_{s}$ are $L_{j}$ and $L_{k}$. If the useful fillet edge points $P_{j i}$ and $P_{k i}$ are not $P_{j i}^{s}$ and $P_{k i}^{s}$, respectively, through point $P_{j i}$ make a line parallel to $E_{k}$ on $F_{s}$ and get the intersection point A (hollow square node) on line $L_{j}$. Similarly, corresponding to $P_{k i}$, we get another intersection point B (hollow square node) on line $L_{k}$. Then the ( $V$-face) on $F_{s}$ corresponding to $V_{i}$ will be two 4 -sided faces $\left(V_{i}, P_{j i}, \mathrm{~A}, W_{s i}\right)$ and $\left(V_{i}, W_{s i}, \mathrm{~B}, P_{k i}\right)$. 


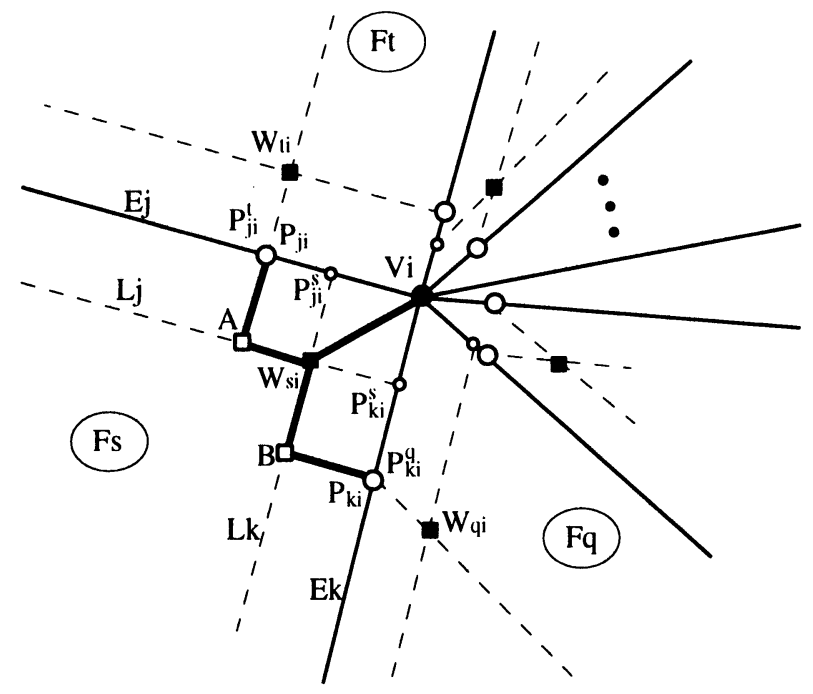

Figure 7 Rule 2. V-face is constructed by two 4-sided faces. Solid square nodes are fillet face points. Hollow circle nodes are the fillet edge points. Small solid circle nodes means there are some faces between $F_{t}$ and $F_{q}$.

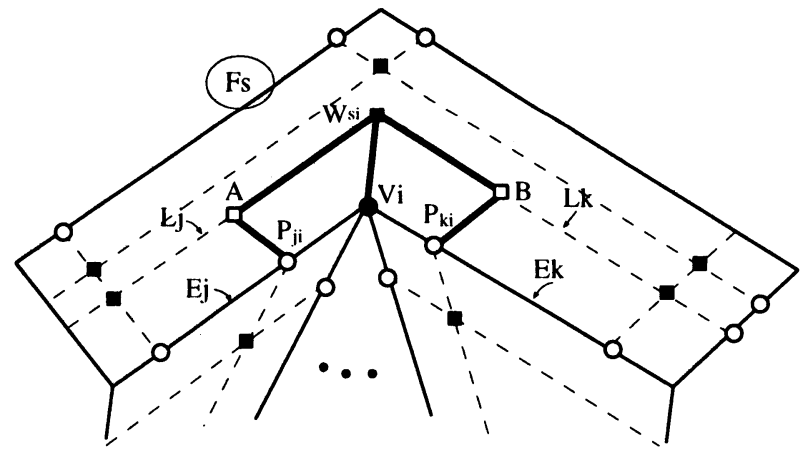

Figure 8 Rule 3 . The fillet subdivision process of a concave point. 


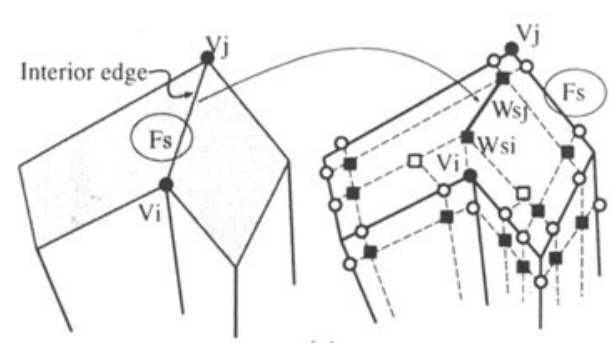

(a)

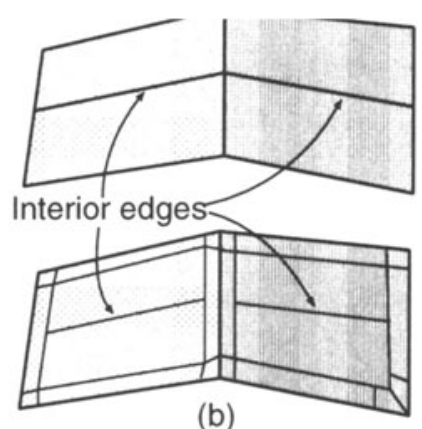

(b)

Figure 9 Rule 4. Fillet subdivision process will not be made on un-boundary edges.

\subsection{RULE 3}

If a vertex $V_{i}$ on a face $F_{s}$ is a concave point, two edges of $F_{s}$ linking $V_{i}$ are $E_{j}$ and $E_{k}$. Corresponding to $E_{j}$ and $E_{k}$, the two fillet lines on $F_{s}$ are $L_{j}$ and $L_{k}$. Because the $V_{i}$ is a concave point on the face $F_{s}$, the fillet edge points $P_{j i}^{s}$ and $P_{k i}^{s}$ do not exist, the fillet edge points $P_{j i}$ and $P_{k i}$ are decided by the fillet lines on other two faces sharing $E_{j}$ and $E_{k}$ with face $F_{s}$, respectively. Similar to the rule 2, through point $P_{j i}$ make a line parallel to $E_{k}$ and get the intersection point $\mathrm{A}$ on $L_{j}$. Similarly we can get intersection point $\mathrm{B}$. Then the $\left(V\right.$-face) on the face $F_{s}$ corresponding to $V_{i}$ is two 4 -sided face $\left(V_{i}, W_{s i}, A, P_{j i}\right)$ and $\left(V_{i}, P_{k i}, B, W_{s i}\right)$. (Referring to Fig.8)

\subsection{RULE 4}

Referring to Fig.9, in some cases, for controlling recursive subdivision surfaces, some faces or concave faces are divided, even on a same plane, this means there are some edges shared by two faces that are on a same plane. In this case, the fillet operations will not be carried out and these edges are called un-boundary edges. Boundary edges are the edges shared by two faces which are not on a same plane. The fillet operations will be done only on the boundary edges. After fillet subdivision, the unboundary edges are linked by new generated fillet face points or the new points on the fillet lines, correspondingly. For example, in Fig.9(a), $F_{s}$ is a face including a concave point $V_{i}$, for controlling the final generated shape, an edge linking $V_{i}$ and $V_{j}$ is added. The fillet operations will not be done on this un-boundary edge. It will be modified by linking $W_{s j}$ and $W_{s i}$. The Fig.9(b) illustrates another case. 


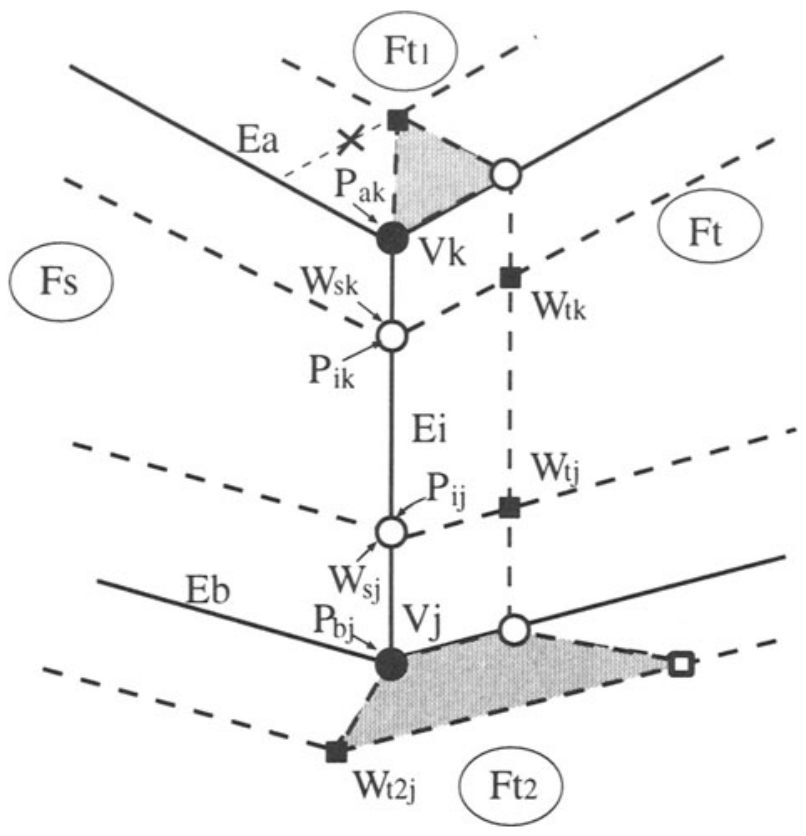

Figure 10 Rule 5. On surface $F_{s}$, the fillet value of edge $E_{i}$ is 0 .

\subsection{RULE 5}

In rule 1 to rule 4 , we assume the fillet values of the edges are not equal to 0 . If the fillet values of some edges are equal to 0 , this means the fillet operations are not carried out on these edges. The edge will be made round by subdivision process. We give rule 5 to deal with this case.

For an edge $E_{i}$ linking vertices $V_{j}$ and $V_{k}$, shared by $F_{s}$ and $F_{t}$, if the fillet value of $E_{i}$ on the face $F_{s}$ equals to 0 . The $V$-face corresponding to $V_{j}$ and $V_{k}$ on the $F_{s}$ will not be generated, the fillet face point $W_{s j}$ and $W_{s k}$ will be on the edge $E_{i}$. The fillet edge points on the edges $E_{a}$ and $E_{b}$ of $F_{s}, P_{a k}$ and $P_{b j}$, will be $V_{k}$ and $V_{j}$, receptively. On two faces $F_{t 1}$ and $F_{t 2}$ that meet at $V_{j}$ and $V_{k}$ and adjoin $F_{t}$ and $F_{s}$, receptively, the $V$-face corresponding to $V_{j}$ and $V_{k}$ will be modified. If the $V$-face is constructed by two 4 -sided faces, the one adjoins $F_{s}$ will not be generated. Only one 4 -sided face(4-sided shadow area in Fig.10) is kept. If the $V$-face is only one 4-sided face, then it will be split into two 3 -sided faces by linking $V_{j}$ and the corresponding fillet face point $\mathrm{W}$ and the one adjoining $F_{s}$ will be deleted. Only one 3-sided face(3-sided shadow area in Fig.10) is kept, referring to Fig.10. If the two fillet values of $E_{i}$ equal to 0 , the 


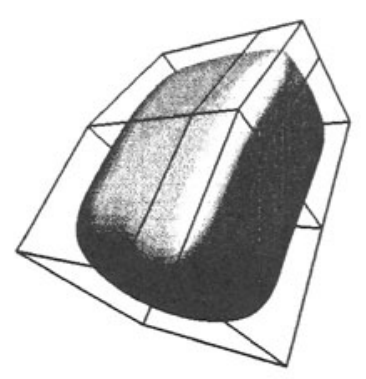

(a)

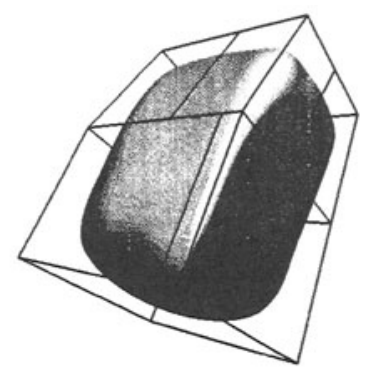

(b)

Figure 11 Rule 5. The two fillet values of an edge are equal to 0 .

$V$-faces of two end points $V_{j}$ and $V_{k}$ will not be generated. Fig.11(a) shows the case that fillet values on both side of an edge are equal each other. Fig.11(b) shows the general case.

\section{EXAMPLES}

In this section, we make fillet operations on some polyhedra with the above rules. For demonstrating the effect of fillet operations, we use Doo-Sabin and Catmull-Clark methods to generate surfaces. Fig.12 illustrates fillet operations on a polyhedron with some vertices having valence four. The fillet values of each edge are different. Fig.12(a) is the original polyhedron. Fig.12(b),(c) are Doo-Sabin surface and Catmull-Clark surface generated form Fig.12(a) after 5 iterations, respectively. Fig.12(d) is the polyhedron after fillet subdivision process. Fig.12(e),(f) are DooSabin surfaces generated from Fig.12(d). Fig.12(g),(h) are CatmullClark surfaces generated from Fig.12(d). Fig.13 illustrates fillet operations on a polyhedron with a vertex having valence six. Fig.14 illustrates filler operations for modeling a mouse. Fig. 15 shows two other examples. Fig.15(c),(d) show two schemes of making fillet operations on same initial polyhedron with different fillet values. Fig.15(g) shows a club. Making comparison between the models generated from original polyhedra and the models generated from the polyhedra modified by fillet operations, we can see the effect of fillet operations clearly. 


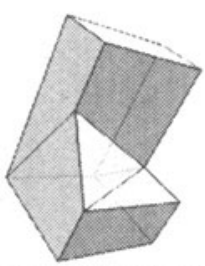

Fig.12(a)Original polyhedron

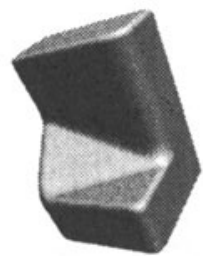

Fig.12(e)Doo/Sabin surface of Fig.12(d)

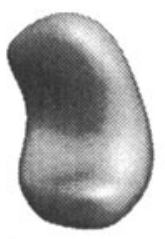

Fig.12(b)Doo/Sabin surface of Fig.12(a)

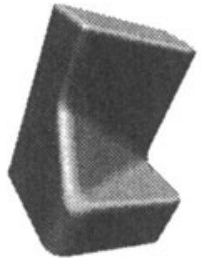

Fig.12(f)Doo/Sabin surface of Fig.12(d)

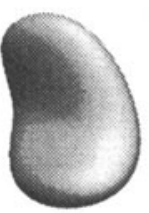

Fig.12(c)Catmull/Clark surface of Fig.12(a)

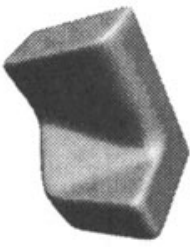

Fig.12(g)Catmull/Clark Fig.12(h)Catmull/Clark surface of Fig.12(d) surface of Fig.12(d)

Fig.12(d)After fillet operations on Fig.12(a)

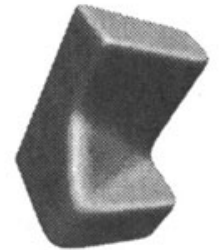

Figure 12. Fillet operation on a polyhedron with some vertices having valence 4.

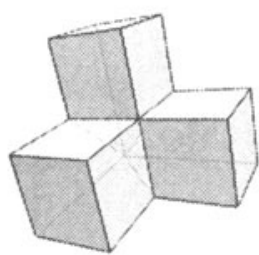

Fig.13(a)Original polyhedron

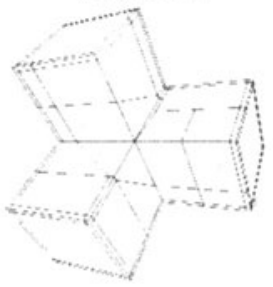

Fig.13(d)After fillet operations on Fig.13(a)

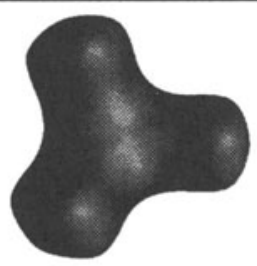

Fig.13(b) Doo/Sabin surface after 5 iterations on Fig.13(a)

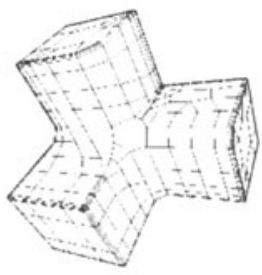

Fig.13(e)Doo/Sabin surface after 1 iteration on Fig.13(d)

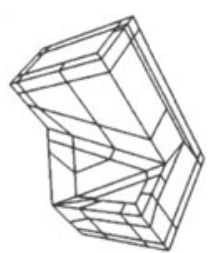

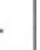

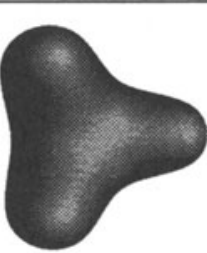

Fig.13(c)Catmull/Clark surface after 5 iterations on Fig.13(a)

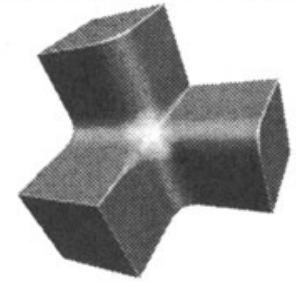

Fig.13(f)Doo/Sabin surface after 5 iterations on Fig.13(d)

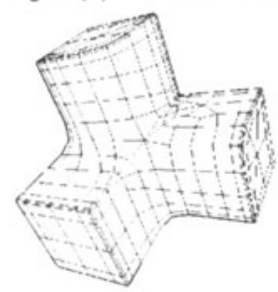

Fig.13(g)Catmull/Clark surface after 1 iteration on Fig.13(d)

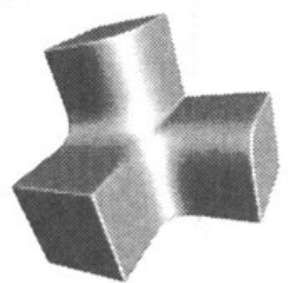

Fig.13(h)Catmull/Clark surface after 5 iterations on Fig.13(d)

Figure 13. Fillet operation on a polyhedron with a vertex having valence 6 . 


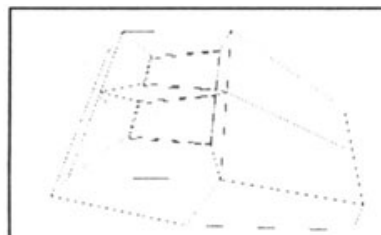

Fig.14(a)Original polyhedron

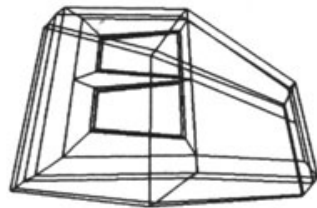

Fig.14(d)After fillet operations on Fig.14(a)

Figure 14. Fillet operations for modeling a mouse.

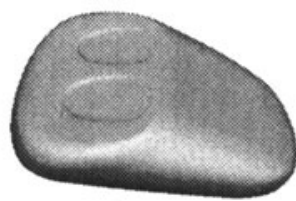

Fig.14(b)Doo/Sabin surface after 5 iterations on Fig.14(a)

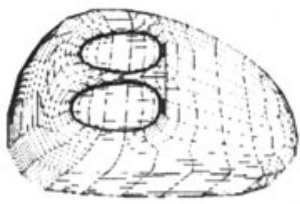

Fig. 14(e)Doo/Sabin surface after 2 iterations on Fig.14(d)

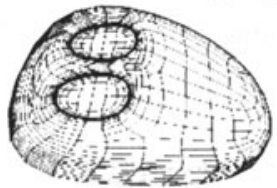

Fig.14(g)Catmull/Clark surface after 2 iterations on Fig.14(d)

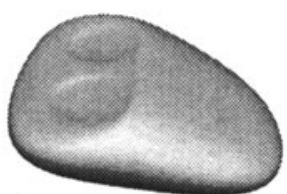

Fig.14(c)Catmul//Clark surface after 5 iterations on Fig.14(a)

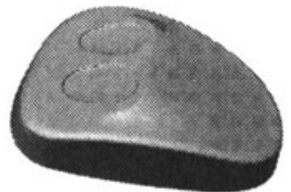

Fig.14(f)Doo/Sabin surface after 5 iterations on Fig.14(d)

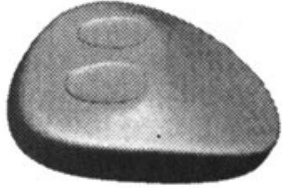

Fig.14(h)Catmull/Clark surface after 5 iterations on Fig.14(d)

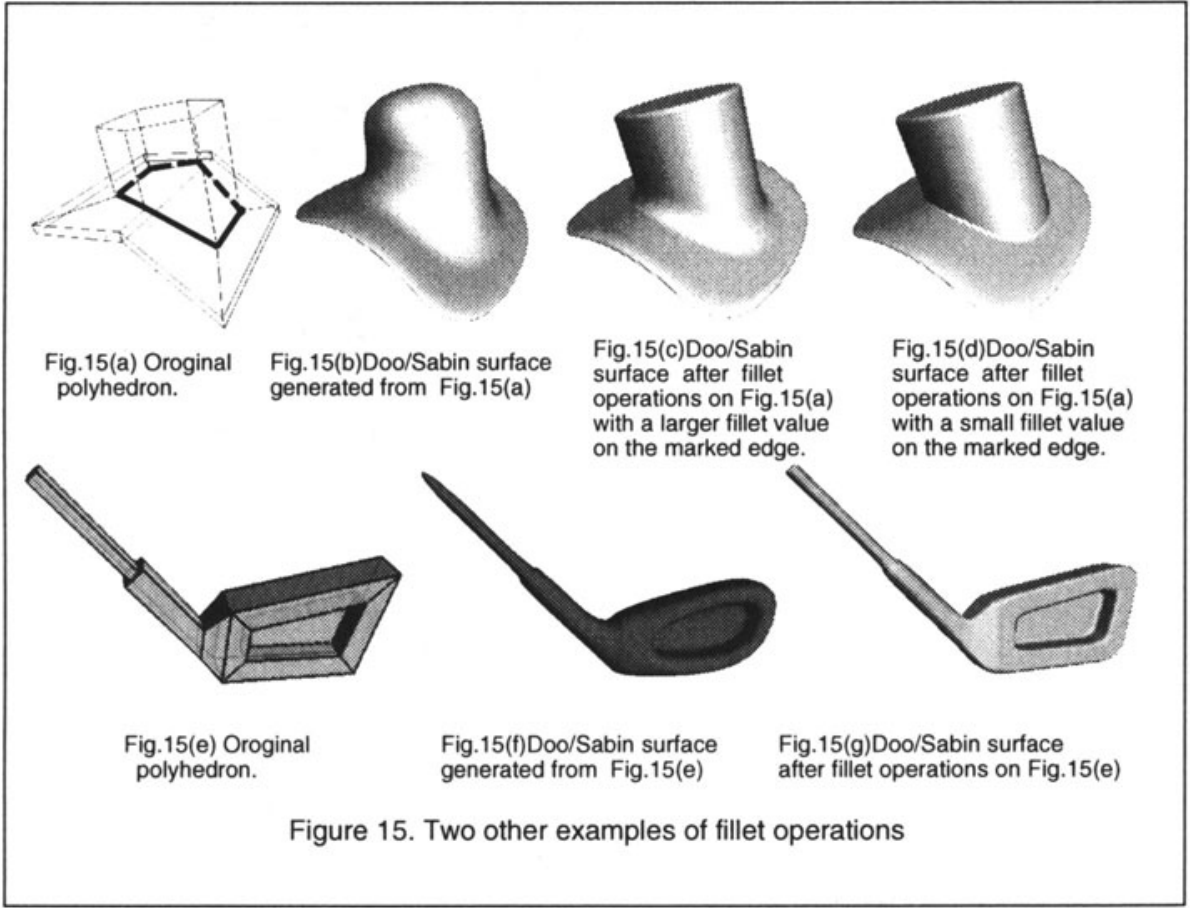




\section{CONCLUSIONS}

In this paper, some rules for implementing the fillet operations with recursive subdivision surfaces are proposed. The polyhedral networks modified by fillet operations can be used to generate both Doo-Sabin surfaces and Catmull-Clark surfaces. The operations are simple and effective. Generally, there is no restriction on the number of edges that meet at one vertex, and fillet values on each edge can be different. It will strengthen the functions of systems that use subdivision method to model surfaces. There are some restrictions here. For example, if the polyhedral networks modified by fillet operations are used by DooSabin method. We can not reach the round areas defined. For solving this problem, we will try to apply Non-Uniform Recursive Subdivision Surfaces[14] to our system in next step.

\section{References}

[1] George Merrill Chaikin: An Algorithm for High-Speed Curve Generation, Computer Graphics and Image Processing, No.3, pp.346$349,(1974)$.

[2] Catmull, E. and Clark, J.: Recursively generated B-spline surfaces on arbitrary topological meshes, Computer Aided Design, Vol.10, No.6, pp.350-355,(Nov.1978).

[3] Doo, D. and Sabin, M.: Behaviour of recursive division surfaces near extraordinary points, Computer Aided Design, Vol.10, No.6, pp.356360,(Nov.1978).

[4] Nasri, Ahmad H.:Polyhedral Subdivision Methods for Free-Form Surfaces, ACM Transaction on Graphics, Vol.6, No.1,pp.29-73, (Jan.1987).

[5] Nasri, A.:C Curve interpolation in recursively generated B-spline surfaces over arbitrary topology, Computer aided Geometric Design, No.14, pp.13-30, (1997).

[6] Nasri, A.:interpolation of Open Curves by Recursive Subdivision Surfaces, Mathematics of Surfaces VII, Information Geometers, pp.173188, (1997).

[7] Reif, U.: A unified approach to subdivision algorithms near extraordinary vertices, Computer aided Geometric Design, No.12,pp.153$174,(1995)$.

[8] Dyn, N., Levin, D., Gregory, J. A.: A Butterfly Subdivision Scheme for Surface Interpolation with Tension Control ACM Transactions on Graphics, Vol.9, No.2, pp.160-169, (Apr.1990) 
[9] Peters, J.: Smoothing Polyhedra Made Easy, ACM Transactions on Graphics, Vol.14.No.2.pp.162-170,(Apr.1995).

[10] Halstead, M., Kass, M. and DeRose, T.: Efficient, Fair Interpolation Using Catmull-Clark Surfaces, Computer Graphics Proceedings, Annual Conference Series,(1993).

[11] T.DeRose, M.Kass and T. Truong: Subdivision Surfaces in Character Animation, Computer Graphics Proceedings, Annual Conference Series, SIGGRAPH'1998, pp.85-94.

[12] Zorin,D., Schroder,P. and Sweldens,W.: Interpolating Subdivision for Meshes with Arbitrary Topology, Computer Graphics Proceedings, Annual Conference Series, pp.189-192,(1996).

[13] Farin, G.: Curves and surfaces for Computer Aided Geometric Design: a practical guide,4th Edition, Academic Press.(1996).

[14] Thomas W.Sederberg, Jianmin Zheng,David Sewell, Malcolm Sabin :Non-Uniform Recursive Subdivision Surfaces, Computer Graphics Proceedings, Annual Conference Series,SIGGRAPH'1998,pp.387394. 